
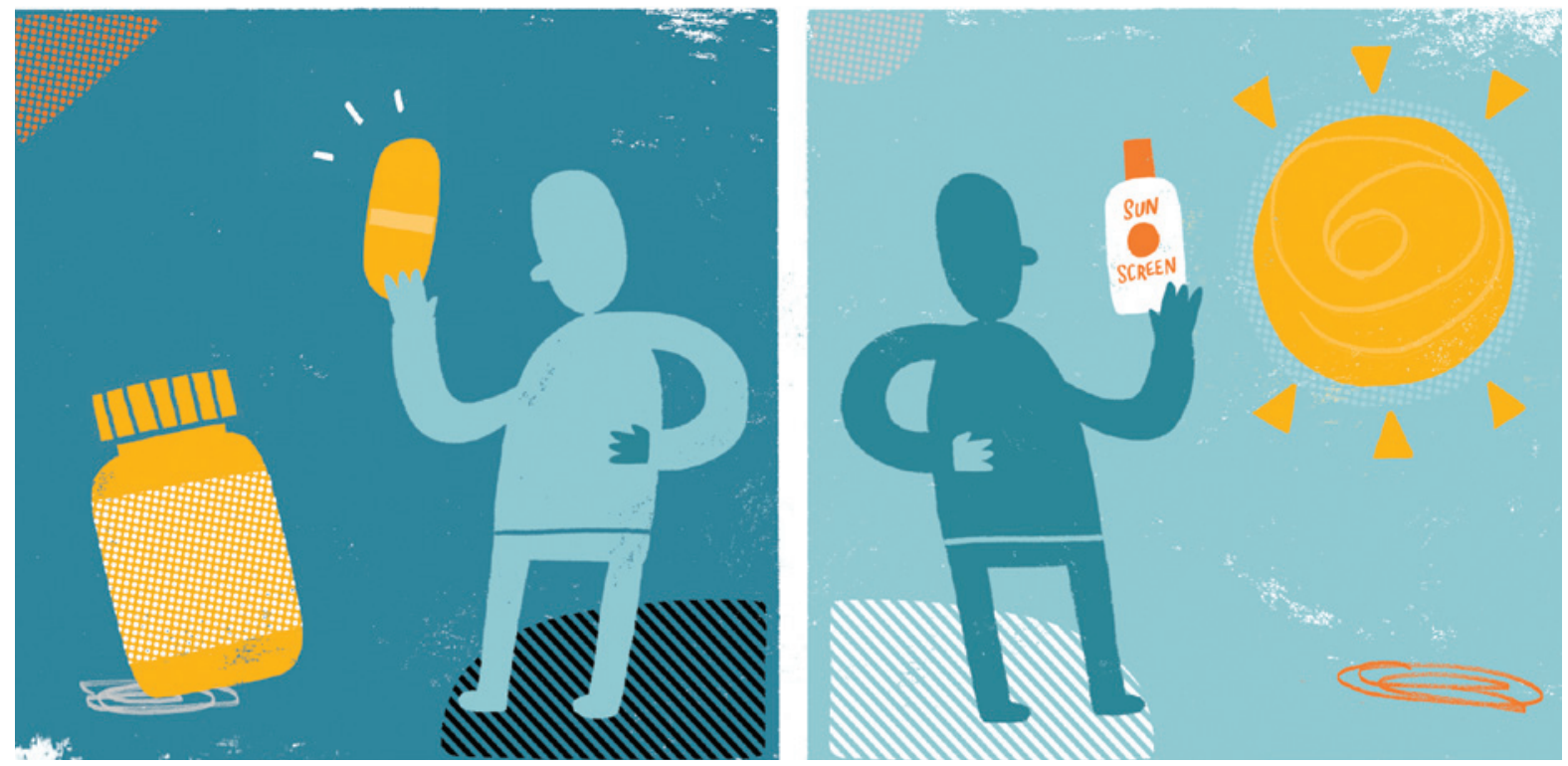

\title{
The vitamin D-lemma
}

\author{
A vociferous debate about vitamin-D supplementation reveals \\ the difficulty of distilling strong advice from weak evidence.
}

BY AMY MAXMEN

$\mathrm{W}$

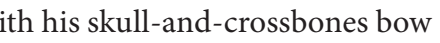
tie tied tight, Clifford Rosen strides to the podium at the Metropoli$\tan$ Bone Club, a meeting of researchers and clinicians in New York City concerned with all things skeletal. He begins by bracing himself: "If you want to ask a question or just yell at me, go ahead," he says. "I'm used to a lot of antagonism, anger, and frustration."

Rosen is director of clinical and translational research at Maine Medical Center Research Institute in Scarborough and is a respected member of the bone-research community. But his role last year on an expert panel to determine how much calcium and vitamin D people need put him at odds with many of his colleagues. In the past few years, vitamin $\mathrm{D}$ has earned a reputation in Western countries for preventing or fighting prostate cancer, cardiovascular disease, multiple sclerosis and about 30 other maladies, leading to advice that most people should be supplementing what the body produces naturally when exposed to sunlight. But in November, the panel, put together by the Institute of Medicine (IOM) - a nonprofit group affiliated with the US National Academy of Sciences - issued a report ${ }^{1}$ that challenged that view. Blood levels of vitamin D need not be as high as many physicians and testing companies had been advocating, it said, and high doses of the vitamin could actually cause harm. Since the report was released, Rosen says he's received about 150 e-mails critical of the panel's decisions. About onethird were downright hateful. "A rehabilitation doctor in Texas threatened to bring me to the board of malpractice to have my licence revoked. People tell me I don't know what I'm doing," he says. "It has become personal."

Much is at stake. By 2009, the amount spent on vitamin-D supplements in the United States had risen tenfold in ten years (see 'Raising the stakes'). Medical practitioners and publichealth officials worldwide look to the IOM for guidance on how to interpret the conflicting claims about vitamin D. Yet several vitamin-D proponents say that the IOM's methods, which involved a systematic review of the literature, were flawed. They have accused the panel of misinterpreting data and over-emphasizing the danger of heavy supplementation. Just last month, the Endocrine Society, a professional association of 14,000 researchers and clinicians based in Chevy Chase, Maryland, released 
guidelines that recommend higher doses than the IOM did².

Why, instead of clearing confusion as was the IOM's goal, has the report sown division and unrest? "The IOM was too definitive in its recommendations," says Michael Holick, an endocrinologist at Boston University School of Medicine in Massachusetts, and an outspoken critic of the IOM panel's conclusions. "Basically, the vitamin-D recommendations are based on low-quality evidence," says Gordon Guyatt, a clinician researcher at McMaster University in Hamilton, Ontario, who has been a consultant on various guidelines. "I think admitting that would have made some of the angst disappear."

Poor data is one reason that the panel did not recommend higher doses, say interested observers. "We are not free to just accept enthusiastic reports, unless they are based on comprehensive, well-characterized data sets," says Paul Coates, director of the Office of Dietary Supplements at the National Institutes of Health in Bethesda, Maryland, one of the agencies that had requested the IOM's evaluation. "I think everyone wants to do the right thing, but I would say that the government is inherently more conservative." The episode demonstrates the difficulty of producing public-health advice from disparate and sometimes feeble evidence. The former panel members have been touring the United States and Europe to defend and explain their methods. That's what brought Rosen to the bone club. "In the past 50 years of IOM reports, this one has received the most visibility - fortunately or unfortunately," he tells the audience.

\section{SYSTEMATIC REVIEW}

Vitamin D's role in promoting bone health through the regulation of calcium is fairly unassailable, but in the past several years, the medical and scientific communities have become preoccupied with how it might prevent chronic disease. Some physicians recommend supplementation of up to 6,000 international units (IU) a day to make up for the time that people spend indoors. This is less than the amount a fair-skinned person without sunblock might make in half an hour of exposure to the midday summer Sun.

In August 2008, the US and Canadian governments asked the IOM for unbiased advice about how much vitamin $\mathrm{D}$ and calcium people need. Rosen and 13 colleagues who were selected to serve on the panel amassed about 1,000 studies on metabolism, vitamin intake and impact on human health. They then ranked the studies by the quality of design and execution. Randomized placebo-controlled studies earned the highest rating. Of roughly 70 such trials, most assessed the effect of vitamin D on falls, fractures and bone quality. About a dozen looked at cancer, cardiovascular disease and diabetes, but because of the way those outcomes were assessed, the panel didn't

\section{RAISING THE STAKES}

Sales of vitamin D in the United States have risen dramatically in the past decade.

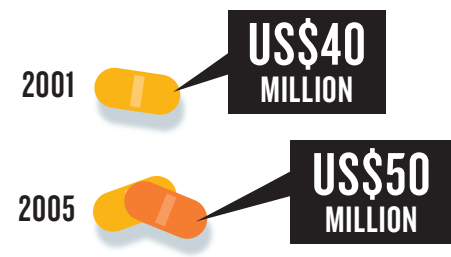

2009

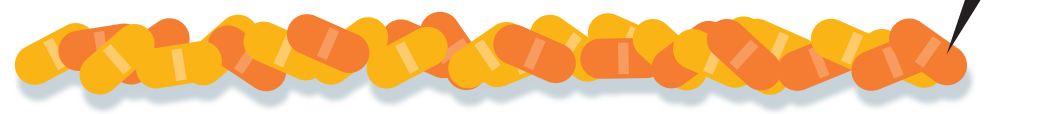

place much trust in the results. Most of the other research has been observational. A 2008 study, for example, reported that men with low levels of vitamin D were more likely to have heart attacks than were those with higher levels ${ }^{3}$, but it couldn't rule out other explanations for the link.

The panel met 8 times over 20 months. Its efforts culminated in a 1,132-page report ${ }^{1}$ concluding that people should aim for blood levels of 50 nanomoles per litre $(\mathrm{nmol} / \mathrm{L})$. This level, the IOM said, can be achieved with $600 \mathrm{IU}$ of vitamin D per day (800 for those older than 70 ) - an amount that doesn't necessarily require supplementation, because many people would get this naturally from Sun exposure, fatty fish and fortified foods. The IOM also stated that reports of widespread deficiency have been exaggerated; the majority of North Americans already have enough vitamin $\mathrm{D}$; and too much of it could be harmful.

Passions ignited immediately. Physicians and alternative-medicine advocates posted websites and Facebook pages declaring the that this might encourage the German government, which does not fortify food, to reconsider the issue.

The IOM was interested in a study Amling had published, in which he had measured bone quality and blood levels of vitamin $\mathrm{D}$ in the bodies of 675 people who had died in good health (for example, in car accidents and suicides) ${ }^{4}$. Amling concluded that an ideal level for the general population would be $75 \mathrm{nmol} / \mathrm{L}$ because everyone above that level had strong bones, and they therefore weren't at a high risk of fractures.

The IOM's mandate was to set the levels that protect most people, but not all. It found that Amling's data supported a $50 \mathrm{nmol} / \mathrm{L}$ threshold (which had been suggested elsewhere in the literature) because at that level, only $1 \%$ of people in the study had weak bones. But Amling says that the IOM made a mathematical mistake: it should have looked at the risk of weak bones in people at or above a certain level, not in the whole population (see 'Denominator dispute'). Instead of dividing

\section{"PEOPLETELL MEIDON'TKNOWWHAT I'M DOING. ITHASBECOMEPERSONAL."}

IOM guidelines flawed. Some claimed that its recommendations were an industry-motivated scheme to keep people in need of prescription drugs and other costly treatments, a theory that Rosen dismisses. And although conspiracy theories tend to be thin on logic or factual substance, scientists and clinicians have raised some legitimate concerns.

\section{STICKY STATS}

Michael Amling, a bone expert at the University Medical Center Hamburg-Eppendorf in Germany is one such critic. He says he was thrilled when Rosen e-mailed him in 2009 to enquire about some of his data. "I wanted to support the work of the IOM," says Amling. He assumed its analysis would conclude that most people were vitamin-D deficient, and the 7 people with weak bones and levels above $50 \mathrm{nmol} / \mathrm{L}$ by all 675 people in the study, he says it should have divided 7 by the 82 individuals with levels above $50 \mathrm{nmol} / \mathrm{L}$. Charles McCulloch, a biostatistician at the University of California, San Francisco, who has no vested interest in vitamin-D thresholds, agrees: the panel should have found that $8.5 \%$ of the population above $50 \mathrm{nmol} / \mathrm{L}$ had weak bones, and therefore according to its goal of allowing no more than $2.5 \%$ of the population to be at risk, Amling's data would support a higher level. "I'm very shocked they made such a basic mathematical mistake," Amling says.

Another researcher whose work received a fair share of the IOM's attention is Heike Bischoff-Ferrari, director of the centre for ageing and mobility at the University of Zurich 
in Switzerland. She published a meta-analysis in 2009 that pooled eight clinical trials testing the ability of vitamin-D supplements to reduce falling in elderly people $e^{5}$. In her analysis, participants who took daily doses of 700-1,000 IU fell less often than those taking a placebo. Doses below 700 IU made no difference.

When the IOM panel came to analyse Bischoff-Ferrari's data, it decided to include different studies. It removed a study ${ }^{6}$ showing a benefit from doses higher than 800 IU because the study had focused on groups of about 20 people, which the panel considered too small. And it added a trial ${ }^{7}$ that BischoffFerrari had excluded because it hadn't been double-blinded. Once the IOM swapped trials in Bischoff-Ferrari's meta-analysis, the evidence showed no benefit from supplementation. Needless to say, Bischoff-Ferrari and others disagree with the IOM's decision.

With no universal criteria to identify which studies ought to be included in meta-analyses, it's hard to say which team selected the most appropriate ones. What is clear, however, is that a lack of high-quality primary research makes these decisions difficult and prone to bias.

Another criticism levelled at the report has to do with the IOM's warning that too much vitamin $\mathrm{D}$ could cause harm. In the only clinical trial claiming risk, elderly women treated with a single 500,000-IU dose of vitamin D annually fell and fractured their bones more often than those in the placebo group ${ }^{8}$. Many researchers find the study ridiculous. "No one absorbs 500,000 IU a day from the Sun, so why would you give that as a supplemental dose?" says Edward Giovannucci, a nutritional epidemiologist at the Harvard School of Public Health in Boston, Massachusetts.

\section{THE DEFENCE}

These are just some of the criticisms that Rosen and other members of the former IOM committee have heard. In response to Amling's charge that the IOM made a mathematical mistake, Rosen maintains that the method the IOM used to calculate $1 \%$ risk is standard procedure for dietary recommendations. The group was asking about a natural population with wide variation in vitaminD levels. He adds that other skeletal studies showed no benefit in increasing the threshold above $50 \mathrm{nmol} / \mathrm{L}$.

With regard to Bischoff-Ferrari's metaanalysis, Rosen stands by the IOM's decision to remove studies with few participants because they are sensitive to random errors; including them, he adds, can exaggerate an erroneous finding.

And JoAnn Manson, an epidemiologist at

Harvard Medical School in Boston, dismisses the notion that the mega-dose trial ought to be ignored: "Within the first three weeks of this trial, when serum levels were at or above $100 \mathrm{nmol} / \mathrm{L}$, there was an increased risk of falls and fractures." This trial contributed to the IOM's anxiety about doses that might raise blood levels to this amount. Specifically, the IOM set an upper dosage limit of 4,000 IU a number it arrived at by taking calculations from various studies. Such precautions are not unexpected, says Guyatt. Health officials put a high value on avoiding recommendations that could prove dangerous over time, he says.

The Endocrine Society's guidelines, which were based on four years of periodic review

DENOMINATOR DISPUTE

a study of 675 people (blue dots), 7 people with vitamin-D blood levels $50 \mathrm{nmol} / \mathrm{L}$ or more had weak bones. The Institute of Medicine (IOM)

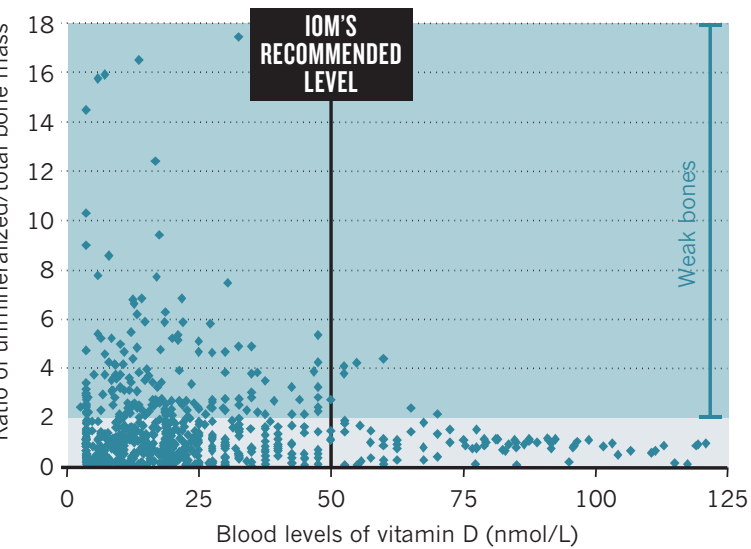

began enrolment for a 5-year, 20,000-person trial to test the effect of supplements on cancer and cardiovascular disease.

Reinhold Vieth, a vitamin-D researcher at the University of Toronto in Canada, calls this demand for huge trials "a cop-out". He says that there is good evidence that higher levels of vitamin D would reduce rates of multiple sclerosis, but a clinical trial to test this would require thousands of people and 30 years. "Saying we need perfect, placebo-controlled trials is denying the plausible evidence we have," Veith says. "At what point do you offer advice?"

Manson urges caution. "We've seen promising correlations before that turned out to be wrong when tested in clinical trials." She offers the example of $\beta$-carotene, which showed promise as a cancer preventative in observational studies but proved dangerous in high-dose trials. "Why are all these lessons of history no longer relevant when it comes to vitamin D?" she asks.

Perhaps IOM panel members underestimated the passion present in the vitamin-D field. Physicians who recommend high doses of vitamin $\mathrm{D}$ might not want to believe that the evidence they have trusted isn't quite up to par. "One thing I wasn't aware of before, is the tremendous pressure from industry and investigators who are tied to their religious belief in vitamin D," says Rosen.

Guyatt says that much of the current fracas could have been avoided if the IOM panel had been a bit more equivocal in its reporting. But Rosen doesn't regret having been dogmatic

of the literature, call people with levels under $50 \mathrm{nmol} / \mathrm{L}$ "vitamin-D deficient", and those with levels between $50 \mathrm{nmol} / \mathrm{L}$ and $72.5 \mathrm{nmol} / \mathrm{L}$ “insufficient”. Insufficiency versus deficiency is not a common distinction in guidelines, says Holick, but it reflects the opinion that people benefit from the higher threshold.

The society's guidelines also offer an 'ideal' level of 100-150 nmol/L for non-skeletal health benefits, which would require 1,5002,000 IU daily, and it advises physicians to monitor vitamin-D levels in healthy people. Quest Diagnostics, a medical-testing corporation headquartered in Madison, New Jersey, that Holick advises, has already begun to implement these deficiency and insufficiency standards over the IOM's, and many physicians are expected to follow suit.

The now-disbanded IOM panel has been formulating a response to clarify how physicians and the public should make sense of the discrepancies in recommendations. The panel's members acknowledge that the case for vitamin $\mathrm{D}$ benefiting general health is not closed, and say that the best way to clarify it is with large clinical trials. Manson recently in the recommendations.

Still, he says that he would have liked to take more time to explain the IOM's methods up front rather than just presenting the bottom line. He suspects that unbiased, systematic reviews such as this one will increasingly come under fire when they lead to hard recommendations. "This is the beginning of a whole new phase," he says. "In the old days of medicine we believed experts, and now we say, show us the data."

Amy Maxmen is a freelance writer in New York City.

1. Institute of Medicine Dietary Reference Intakes for Calcium and Vitamin D (National Academies Press, 2010)

2. Holick, M. F. et al. J. Clin. Endocrinol. Metab. doi:10.1210/jc.2011-0385 (2011)

3. Giovannucci, E., Liu, Y., Hollis, B. W. \& Rimm, E. B. Arch. Int. Med. 168, 1174-1180 (2008).

4. Priemel, M. et al. J. Bone Min. Res. 25, 305-312 (2010).

5. Bischoff-Ferrari, H. A. et al. Br. Med. J. 339, b3692 (2009).

6. Broe, K. E. et al. J. Am. Geriatr. Soc. 55, 234-239 (2007).

7. Law, M., Withers, H., Morris, J. \& Anderson, F. Age Ageing 35, 482-486 (2006)

8. Sanders, K. M. JAMA 303, 1815-1822 (2010). 